\title{
Contribuições da psicologia para repensar o conceito de trabalho decente
}

\author{
Marcelo Afonso Ribeiro ${ }^{1, *}$
}

${ }^{1}$ http:/ /orcid.org/0000-0002-0396-7693 / Universidade de São Paulo (USP), Brasil

Resumo

Trabalho decente é uma concepção objetiva e universalizante baseada em fatores sociais e econômicos proposta pela Organização Internacional do Trabalho (OIT). Diante disso, objetivou-se repensar o conceito de trabalho decente a partir das relevantes contribuições da psicologia internacional e, concomitantemente, brasileira, entendida como um exemplo das experiências do trabalhar no Sul global. Para a respectiva tarefa, levantamos, sistematizamos e analisamos os estudos de trabalho decente a partir da psicologia já publicados através de bases de dados nacionais (Pepsic, Scielo, BVS-Psi, Banco de teses USP, IBICT e CAPES) e internacionais (Scopus, PsycINFO, Proquest e Redalyc), de forma exploratória, analítica e descritiva. Os principais resultados apontaram que trabalho decente é um tema incipiente de investigação na psicologia, e que a psicologia poderia contribuir com os estudos gerais da temática oferecendo uma visão psicossocial e contextualizada para o conceito, na qual sejam articuladas dimensões universais às dimensões singulares, inseridas em determinados contextos sociais e do trabalho.

Palavras-chave: trabalho informal, condições de trabalho, organização do trabalho.

\section{Contributions of psychology to rethinking the decent work concept}

Abstract

Decent work is a generic and universalizing concept based on social and economic factors proposed by the International Labour Organization (ILO). Thus, this study aimed to rethink the concept of decent work through the potential contributions from international and Brazilian psychology, as a global South example. Studies on decent work were extracted from Brazilian (Pepsic, Scielo, BVS-Psi, Banco de teses USP, IBICT, and CAPES) and international (Scopus, PsycINFO, Proquest, and Redalyc) electronic databases, and were organized and analyzed in an exploratory and descriptive fashion. The main results showed that decent work is still characterized as an emerging theme for the field of psychology. Thus, psychology could contribute to this general field of studies by offering a psychosocial and contextualized decent work view. In this view, the concept of decent work should contain universal elements, but these elements should be relativized as a function of the context in which work happens.

Keywords: informal work, working conditions, work organization.

\section{Contribuciones de la psicología para repensar el concepto de trabajo decente}

Resumen

Trabajo decente es un concepto objetivo y universalizador basado en factores sociales y económicos propuesto por la Organización Internacional del Trabajo (OIT). A partir de eso, visamos reflexionar acerca del concepto de trabajo decente desde las posibles contribuciones de la psicología internacional y, concomitantemente, brasileña, comprendida como un ejemplo del trabajar en el Sur global. Para el respectivo trabajo, se realizó un levantamiento, sistematización y análisis de los estudios del trabajo decente ya publicados desde la psicología a través de bases de datos nacionales (Pepsic, Scielo, BVS-Psi, Banco de teses USP, IBICT y CAPES) e internacionales (Scopus, PsycINFO, Proquest y Redalyc), de forma exploratoria, analítica y descriptiva. Los principales resultados apuntaron que el trabajo decente es un tema de investigación incipiente en el área de la psicología. Así, la psicología podría contribuir a este campo de estudios al aportar una visión psicosocial y contextualizada al concepto, donde sean articuladas dimensiones universales a las dimensiones singulares, insertadas en determinados contextos sociales y del trabajo.

Palabras-clave: trabajo informal, condiciones de trabajo, organización del trabajo. 
A importância do trabalho para a vida humana já foi intensamente afirmada e reafirmada pelos mais variados campos do saber e, apesar de constantes questionamentos existentes na literatura especializada (Gorz, 1987; Offe, 1985), sua centralidade ainda é presente no mundo atual, em termos ontológicos, psíquicos, relacionais e sociais, como apontam a maioria dos/as autores/as estudiosos/as do trabalho (p. ex., Antunes, 2012; Blustein, 2011, 2019; Castel, 2005; Spink, 2009).

Castel (2005) diz que o trabalho seria central para a estruturação e a dinâmica social, por isso se constituiria no responsável pela segurança ontológica e pela proteção social no capitalismo construída de duas maneiras. Por um lado, as pessoas que têm propriedade, teriam sua segurança e proteção social garantidas via capital, e, por outro lado, as pessoas que não têm propriedade, teriam sua segurança e proteção social garantidas via trabalho. Castel (2005) define propriedade como propriedade social, ou seja, análoga à propriedade privada e geradora de segurança por um sistema de direitos e obrigações garantidos pelo trabalho (p. ex., a aposentadoria gerada pelo trabalho). Salienta que a grande maioria das pessoas no mundo são trabalhadores/as e não detentores/as de propriedade privada. Segundo dados do Instituto Brasileiro de Geografia e Estatística (IBGE, 2018), no Brasil, 1\% mais rico da população brasileira têm 55\% da renda do país, o que confirma o postulado de Castel (2005).

Além disso, boas condições de trabalho e acesso a trabalhos qualificados e bem remunerados ficam, também, restritos a uma parcela pequena da população, sendo função do Estado gerar políticas que possibilitem a regulação e a estruturação do trabalho como promotor de segurança, não de vulnerabilidade, criando condições para que todos/as possam ter acesso a um trabalho que contenha elementos mínimos para potencialmente poder atender três tipos de satisfação de necessidades humanas básicas. Segundo Blustein (2013, p. 8), o trabalho deve atender "a necessidade de sobrevivência e poder, a necessidade de conexão social, e a necessidade de autodeterminação", se configurando, assim, como um trabalho decente (International Labour Organization, ILO, 1999). Para Duffy, Blustein, Diemer e Autin (2016), as necessidades de sobrevivência e poder se relacionam ao acesso a recursos como alimentação, moradia e capital social; a necessidade de conexão social se relaciona à construção de sentido de vida e de pertencimento social; e, finalmente, a necessidade de autodeterminação se relaciona à possibilidade de controle, escolha e planejamento da vida através da relação estabelecida com os/as outros/as e dos limites e potencialidades psicossociais. Psicossociais, porque seriam constituídas na relação indissociável entre pessoas e contextos (Ribeiro, 2017).

Quanto maior a desigualdade social e a concentração de renda, menos pessoas têm a chance que satisfazer este conjunto de necessidades através do trabalho. Por isso, visando promover melhores condições de trabalho para todos/as, a ILO (1999) postulou o conceito de trabalho decente para embasar ações do Estado com a finalidade de garantir condições dignas de trabalho, segurança, proteção social e direitos garantidos para todos/as em todos os países a partir da lógica do trabalho assalariado (emprego) realizado em nações desenvolvidas.

Um trabalho decente deveria oferecer renda justa, segurança no local de trabalho, proteção social, melhores perspectivas de desenvolvimento pessoal e integração social, respeito pelos direitos fundamentais, liberdade para as pessoas se expressarem, se organizarem e participarem das decisões que afetam suas vidas, e igualdade de oportunidades e tratamento para todas as mulheres e homens (ILO, 1999).

O conceito de trabalho decente emergiu como uma concepção objetiva e universalizante baseada em fatores econômicos e sociais (Ghai, 2002). É, então, um conceito estratégico, não cien- tífico, um discurso genérico produzido por uma instituição internacional (OIT) como instrumento político que, como sublinha Kaplan (2002, p. 180): "opera como modos retóricos e normativos do discurso para convencer os outros a agir", promovendo uma agenda para políticas públicas (Picanço, 2015). Está baseado no modelo do emprego e numa concepção de decente e de dignidade, focado no macrossocial, e forjada a partir do modelo de trabalho dos países desenvolvidos do Norte global (Mattos, 2015).

Diante disso, Deranty e MacMillan (2012), Di Ruggiero, Cohen, Cole e Forman (2015), Ghai (2002) e Proni (2013) têm questionado muito a abrangência e a universalidade do conceito de trabalho decente, principalmente para os países do Sul global que têm como características centrais do mundo do trabalho, para a grande maioria de seus/uas trabalhadores/as, baixa qualidade dos trabalhos, baixas remunerações, alto índice de desemprego e informalidade, e falta de condições de trabalho e de proteção social. Castel (2005) versa que uma grande parte dos/as trabalhadores/as do Sul global vivem sob a égide do individualismo negativo, ou seja, um individualismo por desagregação e falta de referências coletivas de suporte com consequente desproteção social associada, se virando para trabalhar e gerar renda, como pontuou Spink (1996).

Entre as principais questões levantadas pela literatura que vem discutindo o conceito de trabalho decente estão: o trabalho decente deveria ser um conceito universal ou historicamente construída como categoria axiológica aberta (Rosenfield \& Pauli, 2012)? O trabalho decente seria o ponto de partida ou ponto de chegada para as condições de trabalho (Rosenfield \& Pauli, 2012)? O emprego deveria ser o modelo de trabalho decente (Ghai, 2002; Mattos, 2015)? Dever-se-ia criar trabalho decente na economia informal ou eliminar a informalidade para um trabalho decente (Tokman, 2009; Trebilcock, 2005)? Existiria o trabalho decente (no singular) ou haveria versões distintas de trabalhos decentes (no plural) variando em função de contextos distintos (Mattos, 2015)? Há uma lógica ou várias lógicas para alcançar o mesmo fim (Di Ruggiero et al., 2015)? Várias saídas já foram pensadas para estas questões, a maioria baseada no princípio do trabalho decente como um conceito universal, mas que requer contextualização para ser efetivo, pois cada país tem que achar sua própria forma de gerar trabalho decente (Ghai, 2002).

Um movimento recente para compor, juntamente com outros campos do saber, saídas para estes dilemas do conceito de trabalho decente, vem emergindo do campo da psicologia, do qual vários/as autores/as têm defendido a proposta do trabalho decente como uma concepção psicossocial, ou seja, construída de forma contextualizada na relação entre as pessoas e suas realidades sociais (Blustein, Masdonati, \& Rossier, 2017; Blustein, Olle, Connors-Kellgren, \& Diamonti, 2016; Deranty \& MacMillan, 2012; Di Fabio \& Blustein, 2016; Duffy et al., 2016; Ferraro, Pais, Moreira, \& Santos, 2018; Pouyaud, 2016; Ribeiro, Silva, \& Figueiredo, 2016; Ribeiro, Teixeira, \& Ambiel, 2019; Santos, 2019; Spink, 2009). Este movimento tem mais representação no Norte global do que no Sul global e pode responder parcialmente aos dilemas apontados, principalmente ao não lidar, de forma predominante, com contextos de trabalho mais vulnerabilizados.

Burchell, Sehnbruch, Piasna e Agloni (2014), Sehnbruch, Burchell, Agloni e Piasna (2015) e Spink (2009) salientam que o termo trabalho decente tem sido pouco utilizado na literatura acadêmica psicológica, que tem se dedicado, de forma mais intensa, a investigar o trabalho por meio do emprego, não incluindo as formas coexistentes de trabalho, sendo, assim, numa excelente oportunidade para compreender fenômenos como o trabalho informal ou não-regulado, característicos da realidade do Sul global (Proni, 2013; Spink, 2009). Diante disso, o objetivo do presente texto foi repensar o conceito de trabalho decente a partir das potenciais contribui- 
ções da psicologia já existentes na literatura científica especializada, primeiramente internacional e, depois, brasileira, como um exemplo de contextos de trabalho mais vulnerabilizados do Sul global.

As bases de dados consultadas foram Banco de teses USP (Universidade de São Paulo), Banco de teses CAPES (Coordenação de Aperfeiçoamento de Pessoal de Nível Superior), Base Digital de Dissertações e Teses do IBICT (Instituto Brasileiro de Informação em Ciência e Tecnologia), BVS-Psi (Biblioteca Virtual em Saúde - Psicologia Brasil), Pepsic (Portal de Periódicos Eletrônicos de Psicologia), Proquest, PsycINFO, Redalyc, Scielo e Scopus. Foram incluídas, na revisão realizada pelo presente estudo, duas revisões de literatura sobre as publicações de trabalho decente que utilizaram critérios semelhante aos adotados no presente estudo. Na primeira, Grendene Maculan, Campos Junges e Pozzer (2019) estudaram as publicações sobre o tema do trabalho decente na base de dados Web of Science em todas as áreas entre 2008 e 2018, o que nos auxiliou a ampliar nossa revisão de literatura ao incluir uma base não pesquisada. Os/as autores/as destacaram a qual campo do saber cada artigo se filiava e pudemos analisar as produções referentes à psicologia. E, na segunda, Pereira, Santos e Pais (2019) levantaram e analisaram os estudos empíricos do trabalho decente no campo da psicologia e áreas correlatas publicados em língua inglesa entre 2003 e 2017.

Concebemos, então, o conceito de trabalho decente proposto pela OIT (ILO, 1999) como o discurso social normativo (Rossier et al., 2015), padrão discursivo através da qual uma pessoa se adapta à realidade ou cria realidade, sendo uma referência a ser seguida, rejeitada ou transformada. Nosso pressuposto é que o conceito de trabalho decente possa ser reconstruído através da inclusão das contribuições da psicologia em sua elaboração e de contextualização de países do Sul global.

\section{Método}

\section{Revisão de literatura}

O presente estudo se caracterizou como exploratório e descritivo e realizou uma revisão de literatura, na qual consultamos as principais bases de dados científicos nacionais e internacionais onde se encontra a produção científica da área da psicologia. No contexto brasileiro, as bases de dados consultadas foram Banco de teses USP, Banco de teses CAPES, Base Digital de Dissertações e Teses do IBICT, BVS-PSI, Pepsic e Scielo, utilizando como descritores "trabalho decente" e "psicologia" (constando nas palavras-chave, título ou resumo). E, no contexto internacional, as bases de dados consultadas foram Scopus, PsycINFO e Proquest utilizando, primeiramente, como descritores decent work e psychology (igualmente constando nas palavras-chave, título ou resumo) e depois decent work e psychology e Brazil, para identificarmos publicações internacionais com amostras e grupo de participantes do Brasil. Além disso, pesquisamos a Redalyc, utilizando os respectivos descritores em língua espanhola (trabajo decente e psicología) para consultar a existência de produções latino-americanas.

Escolhemos estes descritores que se relacionaram diretamente aos objetivos da presente pesquisa, porque tomamos como princípio que os/as autores/as deveriam ter como eixo de estudo ou análise o trabalho decente a partir da leitura da psicologia. E escolhemos as bases de dados utilizadas, pois nelas se encontram a grande maioria das publicações do campo da psicologia e teríamos uma amostra significativa para a presente pesquisa. Não foi definido nenhum período específico de busca e incluímos todas as publicações encontradas até 2019, visto que os primeiros artigos publicados na psicologia datam de 2004.

\section{Procedimentos}

Fizemos três modalidades de consulta. Primeiramente, consultamos as bases de dados nacionais e identificamos artigos que atendiam aos critérios estabelecidos na pesquisa, ou seja, artigos nos quais os descritores "trabalho decente" e "psicologia" constavam nas palavras-chave, título ou resumo. Em seguida, consultamos as bases de dados internacionais e identificamos artigos que atendiam aos critérios estabelecidos na pesquisa, ou seja, artigos nos quais os descritores decent work e psychology (trabajo decente e psicología) constavam nas palavras-chave, título ou resumo. E, por fim, consultamos as bases de dados internacionais e identificamos artigos que atendiam aos critérios estabelecidos na pesquisa, ou seja, artigos nos quais os descritores decent work e psychology e Brazil (trabajo decente, psicología e Brasil) constavam nas palavras-chave, título ou resumo, para identificarmos publicações internacionais com amostras e grupo de participantes do Brasil.

A seguir, fizemos o levantamento, a sistematização e a análise dos estudos de trabalho decente a partir da psicologia já publicados nacional e internacionalmente; e o levantamento, a sistematização e a análise dos estudos de trabalho decente a partir da psicologia já publicados com amostras e grupo de participantes do Brasil (nacional e internacionalmente). Por fim, destacamos as potenciais contribuições da psicologia para repensar o conceito de trabalho decente, e, em particular, destacamos as potenciais contribuições da psicologia brasileira, como um exemplo de país do Sul global, com contextos e modelos sociais e de trabalho distintos daqueles utilizados para elaborar o conceito de trabalho decente a fim de repensar este conceito a partir de uma compreensão do Sul global.

\section{Análise dos estudos}

Em três etapas, realizamos a análise dos textos completos dos estudos de trabalho decente a partir da psicologia nas publicações identificadas e consultadas, por meio quais organizamos, de forma operacional, tabelas para sistematizar dados quantitativos nas etapas 1 e 2, e realizamos uma análise qualitativa de conteúdo na etapa 3. Na etapa 1, organizamos e apresentamos os dados sistematizados em termos de número e ano das publicações nas bases de dados nacionais, nas bases de dados internacionais e com amostras e grupo de participantes do Brasil (nacional e internacionalmente). $\mathrm{Na}$ etapa 2 , apresentamos dados relativos à cinco dimensões de análise: posição do trabalho decente na pesquisa realizada (estudo do conceito em si, trabalho decente como resultante de outras variáveis estudadas, e trabalho decente como meio de conseguir determinados fins); campo e teoria de base do estudo realizado; distribuição dos estudos entre ensaios teóricos e pesquisas de campo; público estudado na pesquisa de campo; e local da realização do estudo. Por fim, na etapa 3, realizamos duas modalidades de análise de conteúdo. A primeira analisou o conteúdo das publicações internacionais em termos das potenciais contribuições da psicologia para repensar o conceito de trabalho decente; e a segunda analisou o conteúdo das publicações nacionais e internacionais em termos das potenciais contribuições da psicologia brasileira em termos das características locais do que seria um trabalho decente para repensar o conceito.

\section{Resultados}

A apresentação dos resultados se deu em duas partes. $\mathrm{Na}$ primeira apresentamos as sistematizações dos dados relativos à cinco dimensões de análise anteriormente propostas; e, na segunda parte, realizamos duas modalidades de análise de conteúdo das potenciais contribui- 
ções da psicologia para repensar o conceito de trabalho decente.

\section{Sistematização dos Dados Relativos às Dimensões de Aná- lise}

O primeiro estudo achado com foco no trabalho decente fundamentado na psicologia foi publicado em 2004 (Lindsay \& McQuaid, 2004), as pesquisas se ampliaram a partir de 2016 (77,14\% das publicações aconteceram entre 2015 e 2019) e duplicaram em 2018 e 2019 (47,61\% das publicações aconteceram nestes dois anos), conforme vemos na Tabela 1.

Tabela 1

Publicacõoes sobre Trabalbo Decente e Psicologia nas Bases de Dados Internacionais (Ano)

\begin{tabular}{cc}
\hline Período & Frequência $(n, \%)$ \\
\hline Antes de 2015 & $30(28,57 \%)$ \\
$2016-2017$ & $25(23,80 \%)$ \\
$2018-2019$ & $50(47,61 \%)$ \\
\hline
\end{tabular}

Grendene Maculan et al. (2019) realizaram uma revisão de literatura sobre publicações com a temática do trabalho decente e levantaram que a maioria das publicações se concentra no período de 2015 a 2108 (61,89\%), assim como Pereira et al. (2019) que apontaram que o período de 2015 a 2017 concentrou $60,52 \%$ dos artigos publicados com base na psicologia.

Nas bases de dados internacionais pesquisadas (Scopus, PsycINFO e Proquest), a temática do trabalho decente tem sido estudada por vários campos do saber, principalmente o direito, as ciências políticas e as ciências sociais, sendo que a psicologia representa, em média, 8,79\% do total de publicações (Tabela 2). Grendene Maculan et al. (2019) encontraram 349 publicações sobre trabalho decente na base de dados Web of Science, sendo 27 na psicologia $(7,73 \%)$ e a grande maioria dos estudos nas áreas de administração e economia (42,98\%). E Pereira et al. (2019) encontraram 38 publicações da psicologia de um total de 689 publicações $(5,51 \%)$ nas bases de dados B-On and EBESCO host entre 2003 e 2017. Podemos dizer que os/as pesquisadores/as da psicologia são responsáveis por 5 a 10\% das publicações sobre a temática do trabalho decente. Foram encontradas 749 publicações sobre trabajo decente na base de dados Redalyc, mas nenhuma da área da psicologia. É importante marcar que das 144 publicações encontradas com descritores trabalho decente e psicologia 39 eram repetidas, por isso trabalhamos com um total de 105 publicações; e, das 10 publicações encontradas com descritores trabalho decente, psicologia e Brasil, três eram repetidas, por isso trabalhamos com um total de sete publicações.

Tabela 2

Publicações sobre Trabalho Decente e Psicologia nas Bases de Dados Internacionais

\begin{tabular}{cccc}
\hline Bases de dados & $\begin{array}{c}\text { Total de publi- } \\
\text { cações com o } \\
\text { descritor decent } \\
\text { work }\end{array}$ & $\begin{array}{c}\text { Total de publi- } \\
\text { cações com os } \\
\text { descritores decent } \\
\text { work e psychology }\end{array}$ & $\begin{array}{c}\text { Total de publi- } \\
\text { cações com os } \\
\text { descritores decent } \\
\text { work, psychology e } \\
\text { Bražil }\end{array}$ \\
\hline PsycINFO & 90 & $87(96,7 \%)$ & $6(6,67 \%)$ \\
Scopus & 563 & $42(7,46 \%)$ & $3(0,53 \%)$ \\
Proquest & 575 & $15(2,61 \%)$ & $1(0,17 \%)$ \\
\hline
\end{tabular}

Nota. Os números apresentados são o número bruto e o percentual.

A consulta às bases de dados nacionais mostrou a quase inexistência de publicações do campo da psicologia sobre trabalho decente, sendo encontradas apenas duas publicações no Pepsic (Colcerniani, D’Ávila Neto, \& Cavas, 2015; Spink, 2009) e nenhuma publicação no Scielo, na Base Digital de Dissertações e Teses do IBICT, na BVS-Psi, no Banco de teses USP e no Banco de teses CAPES, assim como a América Latina que apresenta o mesmo padrão e não localizamos nenhum estudo na Redalyc.

Com relação à posição do conceito de trabalho decente na pesquisa realizada, 59 realizaram o estudo do conceito em si (56,19\%), 43 tinham o trabalho decente como resultante de outras variáveis estudadas $(40,95 \%)$ e em três estudos o trabalho decente aparecia como meio de conseguir determinados fins $(2,85 \%)$. Os principais conceitos estudados em conjunto com o trabalho decente foram divididos em antecedentes e consequentes. Os principais antecedentes para um trabalho decente foram: diálogo social, ética em gestão de pessoas, governança corporativa, proteção social e sindicalização. E os principais consequentes do trabalho decente foram: bem-estar, qualidade do trabalho, satisfação no trabalho, saúde física e mental e trabalho significativo, com destaque para bem-estar e trabalho significativo que foram os que mais apareceram nas publicações.

Com relação ao campo e teoria de base do estudo realizado, a partir da informação declarada pelos/as próprios/as autores/as das publicações, podemos perceber um predomínio dos campos da orientação profissional e de carreira - OPC com 39 publicações $(37,14 \%)$ e da psicologia organizacional e do trabalho - POT com 32 publicações $(30,47 \%)$ nos estudos do trabalho decente $(67,61 \%$ do total de publicações), complementados pelo campo da Saúde com 10 publicações (9,52\%). É importante marcar a presença da psychology of working theory - PWT proposta por Blustein (2013) e Duffy et al. (2016) do campo da OPC e a humanitarian work psychology apresentada por Carr, MacLachlan e Furnham (2012) do campo da POT, como teorias relevantes para os estudos do trabalho decente, principalmente a PWT que aparece como referência central em 37 do total de publicações (35,23\%), e tem quatro dos/ as cinco autores/as que mais publicam sobre a temática do trabalho decente como proponentes desta teoria (David Blustein, Ryan Duffy, Kelsey Autin e Blake Allan), complementados por Annamaria Di Fabio. Os periódicos que mais publicaram os artigos aqui analisados foram: Journal of Vocational Behavior com 13 publicações (12,38\%), Frontiers in Psychology com 11 publicações (10,47\%) e Journal of Counseling Psychology com 9 publicações (8,57\%).

Com relação à distribuição entre ensaios teóricos e pesquisas de campo, dentre as 105 publicações destacadas como estudos da psicologia, há um equilíbrio entre os dois tipos de publicação com 46 ensaios teóricos $(43,80 \%)$ e 59 trabalhos de campo (56,19\%).

Com relação ao público estudado na pesquisa de campo, basicamente eram trabalhadores/as adultos/as, sendo $22(37,28 \%) \mathrm{em}$ situação de vulnerabilidade (trabalhadores/as informais, minorias étnico-raciais, pessoas com deficiência, trabalhadores/as domésticos/as, imigrantes, desempregados/as, mulheres em situação de violência e empregados/as de baixa renda); 20 (33,89\%) eram trabalhadores/as adultos/as não identificados/as como em situação de vulnerabilidade; 9 (15,25\%) eram estudos de instituições internacionais e empresas; e $6(10,16 \%)$ foram classificados como jovens universitários/as ou do ensino médio. Não foi possível identificar os públicos estudados em duas publicações analisadas (3,38\%).

Os principais locais de realização dos estudos com pesquisa de campo foram os Estados Unidos com 15 publicações $(25,42 \%)$ seguidos de Itália com 6 publicações (10,16\%), Brasil com 5 publicações $(8,47 \%)$ e China com 5 publicações (8,47\%). Canadá, Coréia do Sul, Portugal, Reino Unido e Turquia têm de duas a quatro publicações, enquanto Alemanha, Austrália, França, Ilhas Fiji, Índia, Japão, Nicarágua, Nigéria, Nova Zelândia, Quirquistão, Suiça e Tanzânia tem uma publicação cada um. É importante salientar que a maioria dos estudos realizados foi no Norte global (85 estudos correspondendo a $80,95 \%$ das publicações) e apenas 20 estudos foram conduzidos no Sul global (19,04\%). 
Análise de Conteúdo das Potenciais Contribuições da Psicologia para Repensar o Conceito de Trabalho Decente

Como já indicado, trabalho decente vem se configurando como um conceito emergente estudado no campo da psicologia (Blustein et al., 2017; Grendene Maculan et al., 2019; Pereira et al., 2019). As publicações analisadas apontaram contextos, contribuições e problemas a serem resolvidos em relação ao conceito de trabalho decente. Esta seção analisou apenas os estudos realizados no Norte global, sendo que os estudos do Sul global foram apresentados na seção seguinte a partir do caso de estudos fundamentados na realidade de trabalho do Brasil.

Em termos contextuais, as publicações enfatizaram o déficit de trabalho decente nas amostras e grupos de participantes estudados e desafios para alcançar os objetivos da agenda de trabalho decente, e que a falta de trabalho decente é um sintoma mundial independentemente do nível de desenvolvimento dos países (Di Fabio \& Blustein, 2016; Dodd, Hooley, \& Burke, 2019; Duffy et al., 2016; Pereira et al., 2019).

Várias publicações apontaram mecanismos e estratégias que promoveriam o trabalho decente, como mudanças na governança corporativa (Kubo, 2018) e enfoques humanistas de organização do trabalho (Carr, McAuliffe, \& MacLachlan, 2014), mas que a falta de trabalho decente reduziria a possibilidade de um trabalho significativo (Di Fabio \& Blustein, 2016).

A principal contribuição do conceito de trabalho decente para o campo da psicologia seria conseguir reunir e articular temas relativos ao trabalho que ficam dispersos em estudos específicos (Pereira et al., 2019), como, por exemplo, satisfação no trabalho (Duffy et al., 2019), bem-estar (Autin et al., 2019; Giunchi, Vonthron, \& Ghislieri, 2019; Kubo, 2018) e trabalho significativo (Di Fabio \& Blustein, 2016; Kenny et al., 2016; Lysova, Allan, Dik, Duffy, \& Steger, 2019), buscando uma análise ampliada e mais complexa do trabalho nas mais variadas situações (Blustein et al., 2017).

$\mathrm{E}$ os principais problemas a serem resolvidos em relação ao conceito de trabalho decente seriam que ele é um conceito mais relevante para países desenvolvidos, portanto descontextualizado para parte do mundo (Santos, 2019), ainda é pouco estudado, a maioria dos estudos analisa o conceito parcialmente, há poucos instrumentos de mensuração de componentes do trabalho decente, e há poucas populações distintas estudadas (Blustein et al., 2016; Dodd et al., 2019; Pereira et al., 2019).

Em relação aos estudos do trabalho decente na psicologia, os hot topics nos estudos de trabalho decente não incluem conceitos psicológicos (Grendene Maculan et al., 2019) e a maioria dos estudos propõe ampliar o conceito de trabalho decente, incluindo dimensões psicológicas, mas, em geral, não questionam o conceito em si (Di Fabio \& Kenny, 2016; Ferreira et al., 2019; Masdonati, Schreiber, Marcionetti, \& Rossier, 2019), com algumas exceções (Dodd et al., 2019).

Blustein et al. (2017), em texto do campo da psicologia produzido para a OIT, discutiram o papel da psicologia na agenda de trabalho decente e concluíram que: "uma perspectiva psicológica permite uma melhor compreensão da relação entre o contexto socioeconômico, as diversas condições de trabalho e vida, e o nível geral de saúde e bem-estar das pessoas e a estabilidade das comunidades" (p. 3). Propuseram agendas de pesquisa colaborativa entre a psicologia e a OIT, tendo como eixo central a questão das identidades de trabalho (working identities), entre elas: "Trabalho decente, bem-estar e identidade", "Desenvolvimento de medidas psicológicas de trabalho decente e fatores relacionados", "Pensamento crítico, consciência crítica e desenvolvimento de carreira", "Impacto da renda estável no funcionamento e bemestar psicológico", "Impacto do trabalho decente na vida decente", e "Pesquisa sobre trabalho e bem-estar no Sul global" (p. 10-11).

A última proposta de investigação de Blustein et al. (2017) nos direciona a compreender como as produções do campo da psicologia no Sul global poderiam contribuir para repensar o conceito de trabalho decente. Acolhendo esta recomendação, iremos levantar os principais achados das poucas pesquisas sobre trabalho decente no campo da psicologia brasileira como um exemplo das produções do campo da psicologia no Sul global.

\section{Análise de Conteúdo das Potenciais Contribuições da Psicologia Brasileira para Repensar o Conceito de Trabalho Decente}

A partir das poucas contribuições de estudos com amostras ou grupo de participantes do Brasil, podemos fazer alguns apontamentos a respeito do conceito de trabalho decente. Em primeiro lugar, Ferraro et al. (2017), Ferraro, Moreira, et al. (2018) e Ferraro, Pais, et al. (2018) propõem ampliar o conceito de trabalho decente, incluindo dimensões psicológicas, mas não questionam o conceito em si, diferentemente de Ribeiro et al. (2016) e Spink (2009, 2011). Em segundo lugar, Ribeiro et al. (2016), Ribeiro et al. (2019) e Spink $(2009,2011)$ chamaram atenção, como já indicado nos estudos do Norte global, da problemática do conceito de trabalho decente ser focado no modelo do emprego, não conseguindo dar conta de analisar a informalidade, que é parte constituinte importante do mundo do trabalho no Sul global; e salientaram a importância de um enfoque centrado nos meios de vida (livelihoods) para ampliar o conceito de trabalho decente, principalmente Spink (2011). Em terceiro lugar, os resultados de Ribeiro et al. (2016) em geral, apontaram que os/as trabalhadores/as sem formação superior estudados/as buscam trabalhar de acordo com os princípios recomendados pela OIT, no entanto, esses princípios são construídos na comunidade e não são oferecidos pelo poder público, o que gera formas distintas de trabalho decente.

As redes informais de relacionamento produzem oportunidades de emprego e proteção social, a qualificação ocorre através do aprendizado prático com colegas mais experientes, a oportunidade de continuar trabalhando leva ao sucesso e à segurança e a possibilidade de fazer escolhas e ter controle sobre sua vida se traduz em desenvolvimento pessoal e ocupacional (Ribeiro et al., 2016, p. 1).

Em quarto lugar, os resultados de Ribeiro et al. (2019) assinalaram que nas narrativas de trabalhadores/as urbanos/as de alta qualificação:

Em primeiro lugar, a questão do respeito aparece como elemento fundante de qualquer relação de trabalho. Em segundo lugar, a questão das condições dignas de trabalho que devem ser garantidas tanto por condições objetivas (infraestrutura física e material), quanto psicossociais (bom ambiente relacional, reconhecimento e valorização dos/as trabalhadores/as). Em terceiro lugar, a importância da dimensão psicológica da realização e de um trabalho com sentido para todos/as, que é uma dimensão não sublinhada pela OIT (Ribeiro et al., 2019, p. 238).

Por fim, a necessidade central de que um trabalho para ser considerado decente tenha que articular tanto o desenvolvimento pessoal, quanto social, ou seja, o adjetivo decente estaria vinculado à capacidade que o trabalho teria de articular pessoas e sociedade a fim de construir um mundo melhor, dimensão igualmente não sublinhada pela OIT.

Em síntese, o que os estudos com amostras e grupos de 
participantes brasileiros/as ou ensaios teóricos fundamentados na realidade de trabalho do Brasil pareceram indicar foi que o conceito de trabalho decente tem alguns aspectos universais, entretanto deve ser contextualizado para ser congruente com cada uma das diferentes realidades de trabalho no Sul global, como a literatura já vinha assinalando (Blustein et al., 2017; Ghai, 2002).

\section{Discussão}

O trabalho decente é um novo conceito do campo de estudos do trabalho proposto em 1999 como um conceito estratégico e político por uma instituição internacional reguladora que é a OIT (ILO, 1999). De forma preliminar, o que o conjunto dos estudos analisados indicou é que a falta de trabalho decente é um sintoma mundial independentemente do nível de desenvolvimento dos países (p. ex., Di Fabio \& Blustein, 2016; Pereira et al., 2019).

Por conta da relevância desta constatação, o principal desafio é estudar o conceito de trabalho decente de forma científica (Spink, 2009). As ciências, principalmente as ciências sociais e humanas, dentre elas a psicologia, ainda estão dando os primeiros passos na abordagem científica do conceito de trabalho decente como um al., (2019), e pela revisão e análise realizada no presente estudo. Podemos dizer, então, que os estudos do trabalho decente ainda se encontram em fase de construção em direção a uma consolidação futura, buscando a integração de conhecimentos baseados em evidências para a explicação e gestão do trabalho decente.

Esta conclusão pode ser verificada pelo fato de que, como campo emergente de investigação em geral e, em particular na psicologia, os estudos do trabalho decente tem uma produção mais significativa desde 2015 (77,14\% entre 2015 e 2019), intensificando-se a partir de 2018 (47,61\% em 2018 e 2019), ou seja, é um campo em processo de constituição.

A participação da psicologia neste campo de investigação ainda é incipiente (5 a 10\% das publicações consultadas) e a participação de pesquisas com amostras e grupo de participantes brasileiros/as é menos de 1\%, indicando um campo de pesquisas em aberto a ser explorado. É importante salientar que foram encontrados apenas dois artigos publicados em língua portuguesa em periódicos brasileiros, ambos ensaios teóricos, sendo que os demais foram publicados em língua inglesa em periódicos de outros países, dentre os quais, temos dois com ensaios teóricos e cinco com pesquisas de campo (vide Tabela 3).

Tabela 3

Pesquisas sobre Trabalho Decente com Amostras ou Grupo de Participantes do Brasil

\begin{tabular}{|c|c|c|c|c|c|}
\hline Estudos & $\begin{array}{c}\text { Temática central } \\
\text { estudada }\end{array}$ & Base de dados & $\begin{array}{l}\text { Modalidade da } \\
\text { publicação }\end{array}$ & $\begin{array}{c}\text { Campo de base do } \\
\text { estudo realizado }\end{array}$ & $\begin{array}{l}\text { Público estudado e/ou } \\
\text { analisado }\end{array}$ \\
\hline Spink (2009) & $\begin{array}{l}\text { Trabalho decente e } \\
\text { trabalho informal }\end{array}$ & Pepsic & Ensaio teórico & $\begin{array}{l}\text { Psicologia organizacional } \\
\text { e do trabalho }\end{array}$ & $\begin{array}{c}\text { Trabalhadores/as da } \\
\text { nanoeconomia }\end{array}$ \\
\hline Spink (2011) & $\begin{array}{c}\text { Trabalho decente e papel } \\
\text { do trabalho na vida }\end{array}$ & Scopus & Ensaio teórico & $\begin{array}{c}\text { Psicologia organizacional } \\
\text { e do trabalho }\end{array}$ & $\begin{array}{l}\text { Trabalhadores/as } \\
\text { informais }\end{array}$ \\
\hline $\begin{array}{l}\text { Colcerniani, D’Ávila } \\
\text { Neto e Cavas (2015) }\end{array}$ & $\begin{array}{l}\text { Trabalho decente e a } \\
\text { participação feminina no } \\
\text { mercado de trabalho }\end{array}$ & Pepsic & Ensaio teórico & Teoria da justiça social & Mulheres \\
\hline $\begin{array}{l}\text { Ribeiro, Silva e } \\
\text { Figueiredo (2016) }\end{array}$ & Trabalho decente & PsycINFO & Pesquisa de campo & $\begin{array}{c}\text { Orientação profissional e } \\
\text { de carreira }\end{array}$ & $\begin{array}{l}\text { Trabalhadores/as } \\
\text { adultos/as sem formação } \\
\text { superior }\end{array}$ \\
\hline $\begin{array}{l}\text { Ferraro, Santos, Pais e } \\
\quad \text { Moreira (2017) }\end{array}$ & $\begin{array}{l}\text { Trabalho decente e } \\
\text { motivação no trabalho }\end{array}$ & PsycINFO & Pesquisa de campo & $\begin{array}{c}\text { Psicologia organizacional } \\
\text { e do trabalho }\end{array}$ & Advogados/as \\
\hline $\begin{array}{l}\text { Ferraro, Moreira, Santos, } \\
\text { Pais e Sedmak (2018) }\end{array}$ & $\begin{array}{l}\text { Trabalho decente, } \\
\text { motivação no trabalho e } \\
\text { capital psicológico }\end{array}$ & Scopus e PsycINFO & Pesquisa de campo & $\begin{array}{c}\text { Psicologia organizacional } \\
\text { e do trabalho }\end{array}$ & $\begin{array}{c}\text { Trabalhadores/as do } \\
\text { conhecimento (alta } \\
\text { qualificação) }\end{array}$ \\
\hline $\begin{array}{l}\text { Ferraro, Pais, Moreira e } \\
\quad \text { Santos (2018) }\end{array}$ & $\begin{array}{l}\text { Trabalho decente, } \\
\text { motivação no trabalho e } \\
\text { capital psicológico }\end{array}$ & PsycINFO & Pesquisa de campo & $\begin{array}{c}\text { Psicologia organizacional } \\
\text { e do trabalho }\end{array}$ & $\begin{array}{c}\text { Trabalhadores/as do } \\
\text { conhecimento (alta } \\
\text { qualificação) }\end{array}$ \\
\hline $\begin{array}{l}\text { Ramos, Silva, Bolela e } \\
\text { Andrade (2018) }\end{array}$ & $\begin{array}{l}\text { Trabalho decente como } \\
\text { complementar de } \\
\text { sustentabilidade }\end{array}$ & PsycINFO & Ensaio teórico & Saúde & \\
\hline $\begin{array}{l}\text { Ribeiro, Teixeira e } \\
\text { Ambiel (2019) }\end{array}$ & Trabalho decente & Scopus e PsycINFO & Pesquisa de campo & $\begin{array}{c}\text { Orientação profissional e } \\
\text { de carreira }\end{array}$ & $\begin{array}{l}\text { Trabalhadores/as } \\
\text { adultos/as de alta } \\
\text { qualificação }\end{array}$ \\
\hline
\end{tabular}

todo. Embora podemos constatar a existência de uma longa tradição de investigação das várias dimensões do trabalho decente vistas de forma separada (Blustein et al., 2017; Pereira et al., 2019). Na psicologia, por exemplo, este tem sido o recurso empregado para a transformação do trabalho decente em um conceito científico, ou seja, tentar identificar e analisar conceitos mais consolidados do seu campo de investigação, como motivação no trabalho, bem-estar e trabalho significativo, enquanto variáveis que compõem um trabalho decente, para depois articulá-las e buscar uma explicação mais ampla (Blustein et al., 2016; Dodd et al., 2019; Pereira et al., 2019).

Pereira et al. (2019) afirmaram que o conceito de trabalho decente, ao agregar diferentes componentes do trabalho, seria um conceito integrador que permitiria uma compreensão mais complexa e aprofundada dos fenômenos constituintes do trabalho. Esta etapa ainda não foi alcançada pelas investigações existentes, de acordo com Grendene Maculan et al. (2019) e Pereira et
As duas áreas específicas da psicologia que estão contribuindo de forma mais preponderante para a emergência e instalação de um campo de investigação sobre o trabalho decente, assumindo esta tarefa de transformá-lo de uma proposta político e estratégica para um conceito científico, são o campo da OPC e da POT, juntamente com o campo da saúde. Isto nos parece previsível já que o conceito de trabalho decente lida com o trabalho de forma digna, basicamente realizado por adultos/ as, que pressupõe saúde física e mental e condições de trabalho - temáticas tradicionais de estudo destas áreas. Apesar disso, a liderança do campo da OPC neste movimento de estudos do trabalho decente, principalmente encabeçado por autores/as da PWT, não pareceria tão óbvia a priori, mas demonstra uma transformação do próprio campo da OPC, tradicionalmente dedicado a pensar as primeiras escolhas de carreira da juventude (os/as jovens representam apenas 10,16\% dos perfis estudados). 
No conjunto dos 105 estudos analisados na revisão de literatura proposta, metade se propõe a analisar o conceito de trabalho decente e metade inclui o trabalho decente como uma variável em conjunto com outras ou como resultante de outras variáveis estudadas. Dos estudos que buscaram analisar o conceito de trabalho decente em si temos duas posições.

De um lado, os estudos do Norte global e de parte do Sul global propõem ampliar o conceito de trabalho decente, incluindo dimensões psicológicas, mas, em geral, não questionam o conceito em si, o que nos leva a concluir que concordam com a lógica do emprego como modelo para o trabalho. Nossa hipótese é que têm um mercado de trabalho onde o emprego (ou variações do emprego, como o modelo de tempo parcial, teletrabalho ou trabalho intermitente) é predominante ou estudaram amostras ou grupo de participantes dos quais o emprego é o modelo de vínculo predominante nos contextos de trabalho.

Do outro lado, temos alguns estudos do Sul global que questionam o conceito de trabalho decente proposto pela OIT, justamente por ele não incluir as várias formas de trabalho existentes em contextos, como o Brasil, para além do modelo do emprego, baseando-se, principalmente, no fato de que, historicamente, por exemplo, parte do mercado de trabalho no Brasil é constituído de trabalho informal ou não-regulado (Antunes, 2012; Proni, 2013). Segundo o IBGE (2020), em 2020 o Brasil tinha $41,1 \%$ de trabalhadores/as realizando atividades consideradas informais ou não-reguladas. É importante salientar que Dodd et al. (2019), apesar de conduzir um estudo no Reino Unido no Norte global, trazem uma preocupação clara com a contextualização do conceito para que ele seja efetivo para todos/as e questionam o modelo proposto pela OIT focado no emprego.

Aqui, portanto, podemos retomar as várias questões enunciadas na introdução sobre a adoção do emprego como modelo para o trabalho decente, se o emprego seria a única forma possível de oferecer um trabalho decente e as demais seriam precárias, e se deveríamos criar trabalho decente na economia informal ou eliminar a informalidade para um trabalho decente. Em resposta a estas perguntas, parte das publicações consultadas do Sul global e, mais especificamente do Brasil (estudo de caso da presente revisão de literatura), entre elas Ribeiro et al. (2016), Ribeiro et al. (2019) e Spink (2009, 2011), afirmou que, em contextos onde o emprego nunca foi predominante, existiria versões distintas de trabalhos decentes (trabalhos decentes no plural) variando em função de contextos distintos, como apontaram teoricamente Di Ruggiero et al. (2015), Mattos (2015), Tokman (2009) e Trebilcock (2005).

Neste sentido, as pesquisas se focaram em apontar como estudar o trabalho decente e o que seria um trabalho decente nos mais variados contextos sociais e de trabalho, e não como ele não é presente em vários contextos de trabalho a partir de um conceito normativo definido a priori, como é o caso do conceito de trabalho decente proposto pela OIT (ILO, 1999), que funcionaria como o discurso social normativo (Rossier et al., 2015). Assim, as poucas pesquisas brasileiras encontradas na revisão de literatura assinalaram a necessidade de se ter um padrão discursivo, como o conceito de trabalho decente da OIT, mas que deve ser transformado em função de contextos distintos de trabalho. Esta transformação incluiria tanto a pluralidade de experiências dos/ as trabalhadores/as, quanto das organizações e condições do trabalho em dado contexto, o que parece confirmar nosso pressuposto inicial de que o conceito de trabalho decente deveria ser reconstruído através da inclusão das contribuições da psicologia em sua elaboração e de contextualização de países do Sul global.

\section{Conclusões}

O presente texto visou repensar o conceito de trabalho decente a partir das potenciais contribuições da psicologia já existentes na literatura científica internacional e brasileira. Primeiramente, é importante salientar que o trabalho decente é um fenômeno recente de investigação, está em fase de construção como campo de pesquisa e tem uma concentração muito grande das pesquisas nos campos do direito, das ciências políticas e das ciências sociais, de pesquisadores/as do Norte global, com uma contribuição modesta do campo da psicologia e quase inexistente da psicologia do Sul global. Os achados da pesquisa possibilitaram três conclusões centrais resumidas em três potenciais contribuições da psicologia para a agenda de investigações do trabalho decente como um conceito científico, a saber: o trabalho decente se mostrou como um conceito integrador ao conseguir reunir e articular temas relativos ao trabalho que ficam dispersos em estudos específicos; deve incluir uma visão psicossocial em sua análise; e o trabalho decente deve ser um conceito universal, mas contextualizado para poder fazer sentido e poder oferecer contribuições concretas para todos/as os/as trabalhadores/as.

Tanto a universalidade, quanto a singularidade do conceito de trabalho decente são importantes e, neste sentido, corroboramos com um princípio proposto pelo campo do direito que nos parece pertinente para pensar o conceito de trabalho decente em contextos sociais e de trabalho, como a realidade do Brasil, de que "o universal não é o ponto de partida, mas sim o de chegada" (Rosenfield \& Pauli, 2012, p. 322), ou seja, tanto elementos contextuais, quanto elementos universais são de suma importância para um trabalho decente.

Assim, a revisão de literatura realizada nos permitiu constatar que a contribuição da proposta conceitual da OIT foi muito importante, mas que o conceito de trabalho decente pode ser repensado a partir das potenciais contribuições da psicologia, visando incluir a dimensão psicossocial na concepção de trabalho decente e repensar sua conceituação a partir de contextos de trabalho mais vulnerabilizados do Sul global.

\section{Referências}

Antunes, R. (2012). O caracol e sua concha: Ensaios sobre a nova morfologia do trabalho. São Paulo, SP: Boitempo.

Autin, K. L., Duffy, R. D., Blustein, D. L., Gensmer, N. P., Douglass, R. P., England, J. W., \& Allan, B. A. (2019). The development and initial validation of need satisfaction scales within the psychology of working theory. Journal of Counseling Psychology, 66(2), 195-209. https://doi.org/10.1037/cou0000323

Blustein, D. L. (2011). A relational theory of working. Journal of Vocational Behavior, 79(1), 1-17. https://doi.org/10.1016/j.jvb.2010.10.004

Blustein, D. L. (2013). The psychology of working: A new perspective for a new era. Em D. L. Blustein (Ed.), The Oxford handbook of the psychology of working (pp. 3-18). New York, NY: Oxford University Press.

Blustein, D. L. (2019). The importance of work in an age of uncertainty: The eroding work experience in America. Oxford, UK: Oxford University Press.

Blustein, D., Masdonati, J., \& Rossier, J. (2017). Psychology and the International Labor Organization: The role of psychology in the decent work agenda. Retrieved from http://www.ilo.org/global/research/publications/WCMS 561013/lang-en/index.htm

Blustein, D. L., Olle, C., Connors-Kellgren, A., \& Diamonti, A. J. (2016). Decent work: A psychological perspective. Frontiers in Psychology, 7(407), 1-14. https://doi.org/10.3389/fpsyg.2016.00407

Burchell, B., Sehnbruch, K., Piasna, A., \& Agloni, N. (2014). The quality of employment and decent work: Definitions, methodologies, and ongoing debates. Cambridge Journal of Economy, 38(2), 459-477. https://doi. org $/ 10.1093 / \mathrm{cje} / \mathrm{bet} 067$

Carr, S. C., MacLachlan M., \& Furnham A. (Eds). (2012). Humanitarian work. psychology. London, UK: Palgrave Macmillan.

Carr, S. C., McAuliffe, E., \& MacLachlan, M. (2014). Servants of empowerment. Em W. Reichman (Ed.), Industrial and organizational psychology help the vulnerable: Serving the underserved (pp. 143-163). London, UK: Palgrave Macmillan. https://doi.org/10.1057/9781137327734 9 
Castel, R. (2005). A insegurança social: O que é ser protegido? Petrópolis, RJ: Vozes.

Colcerniani, C. B., D’Ávila Neto, M. I., \& Cavas, C. de S. T. (2015). A participação das mulheres no mercado de trabalho sob a perspectiva da teoria da justiça social de Nancy Fraser e dos conceitos relativos ao trabalho decente. Cader nos de Psicologia Social do Trabalho, 18(2), 169-180. https://doi.org/10.11606/ issn.1981-0490.v18i2p169-180

Deranty, J.-P., \& MacMillan, C. (2012). The ILO's decent work initiative: Suggestions for an extension of the notion of "decent work". Journal of Social Philosophy, 43(4), 386-405. https://doi.org/10.1111/iosp.12003

Di Fabio, A., \& Blustein, D. L. (2016). Editorial: From meaning of working to meaningful lives: The challenges of expanding decent work. Frontiers in Psychology, 7(1119), 1-2. https://doi.org/10.3389/fpsyg.2016.01119

Di Fabio, A., \& Kenny, M. E. (2019). Decent work in Italy: Context, conceptualization, and assessment. Journal of Vocational Behavior, 110, 131-143. https:// doi.org/10.1016/i.jvb.2018.10.014

Di Ruggiero, E., Cohen, J. E., Cole, D. C., \& Forman, L. (2015). Competing conceptualizations of decent work at the intersection of health, social and economic discourses. Social Science \& Medicine, 133, 120-127. https://doi. org/10.1016/j.socscimed.2015.03.026

Dodd, V., Hooley, T., \& Burke, C. (2019). Decent work in the UK: Context, conceptualization, and assessment. Journal of Vocational Behavior, 112, 270281. https://doi.org/10.1016/i.jvb.2019.04.002

Duffy, R. D., Blustein, D. L., Diemer, M. A., \& Autin, K. L. (2016). The psychology of working theory. Journal of Counseling Psychology, 63(2), 127-148. https://doi.org/10.1037/cou0000140

Duffy, R. D., Kim, H. J., Gensmer, N. P., Raque-Bogdan, T. L., Douglass, R. P. England, J. W., \& Buyukgoze-Kavas, A. (2019). Linking decent work with physical and mental health: A psychology of working perspective. Journal of Vocational Behavior, 112, 384-395. https://doi.org/10.1016/i.jvb.2019.05.002

Ferraro, T., Moreira, J. M., Santos, N. R., Pais, L., \& Sedmak, C. (2018). Decent work, work motivation and psychological capital: An empirical research. Work: Journal of Prevention, Assessment \& Rehabilitation, 60(2), 339-354. https://doi.org/10.3233/WOR-182732

Ferraro, T., Pais, L., Moreira, J. M., \& Santos, N. R. D. (2018). Decent work and work motivation in knowledge workers: The mediating role of psychological capital. Applied Research in Quality of Life, 13(2), 501-523. https://doi.org/10.1007/s11482-017-9539-2

Ferraro, T., Santos, N. R., Pais, L., \& Moreira, J. M. (2017). Decent work and work motivation in lawyers: An empirical research. Revista Psicologia: Organizações e Trabalho, 17(4), 192-200. http://doi.org/10.17652/rpot/2017.4.13908

Ferreira, J. A., Haase, R. F., Santos, E. R., Rabaça, J. A., Figueiredo, L., Hemami, H. G., \& Almeida, L. M. (2019). Decent work in Portugal: Context, conceptualization, and assessment. Journal of Vocational Behavior, 112, 77-91. http://doi.org/10.1016/i.jvb.2019.01.009

Ghai, D. (2002). Decent work: Universality and diversity. Geneva, Switzerland: International Labour Organization - ILO.

Giunchi, M., Vonthron, A. M., \& Ghislieri, C. (2019). Perceived job insecurity and sustainable wellbeing: Do coping strategies help?. Sustainability, 11(3), 1-18. https://doi.org/10.3390/su11030784

Gorz, A. (1987). Adeus ao proletariado. Rio de Janeiro, RJ: Forense-Universitária.

Grendene Maculan, C., Campos Junges, V., \& Pozzer, P. H. R. (2019). Trabalho decente: Estudo das publicações sobre o tema na base web of science. Observatorio de la Economía Latinoamericana. Recuperado de https://www.eumed.net/ rev/oel/2019/09/estudo-publicacoes-science.html

Instituto Brasileiro de Geografia e Estatística (IBGE, 2018). Pesquisa Nacional por Amostra de Domicilios Contínua. Recuperado de http://www.ibge.gov.br/ home/estatistica/indicadores/trabalhoerendimento/pnad continua

Instituto Brasileiro de Geografia e Estatística (IBGE, 2020). Pesquisa Nacional por Amostra de Domicílios Contínua. Recuperado de http://www.ibge.gov.br/ home/estatistica/indicadores/trabalhoerendimento/pnad continua

International Labour Organization (ILO, 1999). Report on decent work. Retrieved from http://www.ilo.org/public/english/standards/relm/ilc/ilc87/rep-i. $\underline{\mathrm{htm}}$

Kaplan, T. (2002). Reading policy narratives: Beginnings, middles and ends. In F. Fischer \& J. Forestor (Eds.), The argumentative turn in policy analysis and planning (pp. 167-185). Durham, UK: Duke University Press.

Kenny, M. E., Catraio, C., Bempechat, J., Minor, K., Olle, C., Blustein, D. L., \& Seltzer, J. (2016). Preparation for meaningful work and life: Urban high school youth's reflections on work-based learning 1-year post-graduation. Frontiers in Psychology, 7(286). https://doi.org/10.3389/fpsyg.2016.00286

Kubo, K. (2018). The effect of corporate governance on firms' decent work policies in Japan. Asia Pacific Journal of Human Resources, 56(4), 450-473. https://doi.org/10.1111/1744-7941.12176

Lindsay, C., \& McQuaid, R. W. (2004). Avoiding the "Mcjobs": Unemployed job seekers and attitudes to service work. Work, Employment and Society, 18(2), 297-319. https://doi.org/10.1177/09500172004042771

Lysova, E. I., Allan, B. A., Dik, B. J., Duffy, R. D., \& Steger, M. F. (2019). Fostering meaningful work in organizations: A multi-level review and integration. Journal of Vocational Behavior, 110(Part B), 374-389. https://doi. org/10.1016/i.jvb.2018.07.004
Masdonati, J., Schreiber, M., Marcionetti, J., \& Rossier, J. (2019). Decent work in Switzerland: Context, conceptualization, and assessment. Journal of Vocational Behavior, 110, 12-27. https://doi.org/10.1016/i.jvb.2018.11.004

Mattos, M. B. (2015). Conquistas sociais a partir da promoção do trabalho decente no Brasil. Revista Direito do Trabalho, Politica e Sociedade, 1, 252-275. Recuperado de http://revista91.hospedagemdesites.ws/index.php/rdtps/ article/view/12/12

Offe, C. (1985). Capitalismo desorganizado. São Paulo, SP: Brasiliense.

Pereira, S., Santos, N., \& Pais, L. (2019). Empirical research on decent work: A literature review. Scandinavian Journal of Work and Organizational Psychology, 4(1), 1-15. https://doi.org/10.16993/sjwop.53

Picanço, F. S. (2015). Juventude e trabalho decente no Brasil - Uma proposta de mensuração. Caderno CRH, 28(75), 569-590. http://doi.org/10.1590/S0103$\underline{49792015000300008}$

Pouyaud, J. (2016). For a psychosocial approach to decent work. Frontiers in Psychology, 7(422). https://doi.org/10.3389/fpsyg.2016.00422

Proni, M. W. (2013). Trabalho decente e vulnerabilidade ocupacional no Brasil. Economia \& Sociedade, 22, 825-854. http://doi.org/10.1590/S010406182013000300009

Ramos, S. B., Silva, J. P., Bolela, C. A., \& Andrade, M. (2018). Prediction of human development from environmental indicators. Social Indicators Research, 138(2), 467-477. https://doi.org/10.1007/s11205-017-1693-2

Ribeiro, M. A. (2017). Psicossocial: Continuum ontológico do processo relacional. Em N. Silva Jr. \& W. Zangari (Orgs.), A psicologia social e a questão do bifen (pp. 263-277). São Paulo, SP: Edgard Blücher.

Ribeiro, M. A., Silva, F. F., \& Figueiredo, P. M. (2016). Discussing the notion of decent work: Senses of working for a group of Brazilian workers without college education. Frontiers in Psychology, 7(207). https://doi.org/10.3389/ fpsyg.2016.00207

Ribeiro, M. A., Teixeira, M. A. P., \& Ambiel, R. A. M. (2019). Decent work in Brazil: Context, conceptualization, and assessment. Journal of Vocational Behavior, 112, 229-240. https://doi.org/10.1016/j.jvb.2019.03.006

Rosenfield, C. L., \& Pauli, J. (2012). Para além da dicotomia entre trabalho decente e trabalho digno: Reconhecimento e direitos humanos. Caderno CRH, 25(65), 319-329. http://doi.org/10.1590/S0103-49792012000200009

Rossier, J., Nota, L., Dauwalder, J.-P., Duarte, M. E., Guichard, J., Savickas, M. L., ... van Vianen, A. E. M. (2015). Conclusion. Em L. Nota \& J. Rossier (Eds.), Handbook of Life Design (pp. 285-290). Boston, MA: Hogrefe.

Santos, N. R. (2019). Decent work expressing universal values and respecting cultural diversity: Propositions for intervention. Psychologica, 62(1), 233-250. https://doi.org/10.14195/1647-8606 62-1 12

Sehnbruch, K., Burchell, B., Agloni, N., \& Piasna, A. (2015). Human development and decent work: Why some concepts succeed and others fail to make an impact. Development and Change, 46(2), 197-224. https://doi. org $/ 10.1111 /$ dech.12149

Spink, P. K. (1996). Organização como fenômeno psicossocial: Notas para uma redefinição da psicologia do trabalho. Psicologia \& Sociedade, 8(1), 174-192.

Spink, P. K. (2009). Micro cadeias produtivas e a nanoeconomia: Repensando o trabalho decente. Cadernos de Psicologia Social do Trabalho, 12(2), 227-241. https://doi.org/10.11606/issn.1981-0490.v12i2p227-241

Spink, P. K. (2011). Whatever happened to work: From the centrality of shoes, ships and sealing-wax to the problems posed by flying pigs. Athenea Digital (Revista de Pensamiento e Investigación Social), 11(3), 3-24. Retrieved from https: / / atheneadigital.net/issue/viewFile/v11-n3/vol-11-n-3-pdf\#page =9

Tokman, V. (2009). Flexissegurança com informalidade: Opções e restrições. Em J. Weller (Org.), O novo cenário laboral latino-americano: Regulação, proteção e politicas ativas nos mercados de trabalho (pp. 183-206). Santiago, Chile: United Nations/Ministry of Foreign Affairs of Denmark.

Trebilcock, A. (2005). Decent work and the informal economy. Helsinki, Finland: World Institute for Development Economics (UNU-WIDER).

\section{Informações sobre o autor:}

\section{Marcelo Afonso Ribeiro}

Av. Prof. Mello Moraes, 1721. Cidade Universitária/São Paulo

(SP), Brasil. CEP 05508-030.

E-mail: marcelopsi@usp.br 